\title{
Differentiating novel coronavirus pneumonia from general pneumonia based on machine learning
}

\author{
Chenglong Liu ${ }^{1,2+}$, Xiaoyang Wang ${ }^{3 \dagger}$, Chenbin Liu ${ }^{4}$, Qingfeng Sun ${ }^{5}$ and Wenxian Peng ${ }^{2 *}$
}

${ }^{*}$ Correspondence:
pengwx@sumhs.edu.cn
${ }^{\dagger}$ Chenglong Liu and
Xiaoyang Wang equally
contributed as the first
authors
${ }^{2}$ College of Medical
Imaging, Shanghai University
of Medicine and Health
Sciences, Shanghai 201318,
China
Full list of author information
is available at the end of the
article

${ }^{*}$ Correspondence: pengwx@sumhs.edu.cn Xiaoyang Wang equally contributed as the first authors

${ }^{2}$ College of Medica of Medicine and Health Sciences, Shanghai 201318 article

\begin{abstract}
Background: Chest CT screening as supplementary means is crucial in diagnosing novel coronavirus pneumonia (COVID-19) with high sensitivity and popularity. Machine learning was adept in discovering intricate structures from CT images and achieved expert-level performance in medical image analysis.

Methods: An integrated machine learning framework on chest $\mathrm{CT}$ images for differentiating COVID-19 from general pneumonia (GP) was developed and validated. Seventy-three confirmed COVID-19 cases were consecutively enrolled together with 27 confirmed general pneumonia patients from Ruian People's Hospital, from January 2020 to March 2020. To accurately classify COVID-19, region of interest (ROI) delineation was implemented based on ground-glass opacities (GGOs) before feature extraction. Then, 34 statistical texture features of COVID-19 and GP ROI images were extracted, including 13 gray-level co-occurrence matrix (GLCM) features, 15 gray-level-gradient co-occurrence matrix (GLGCM) features and 6 histogram features. High-dimensional features impact the classification performance. Thus, ReliefF algorithm was leveraged to select features. The relevance of each feature was the average weights calculated by ReliefF in $n$ times. Features with relevance larger than the empirically set threshold $T$ were selected. After feature selection, the optimal feature set along with 4 other selected feature combinations for comparison were applied to the ensemble of bagged tree (EBT) and four other machine learning classifiers including support vector machine (SVM), logistic regression (LR), decision tree (DT), and K-nearest neighbor with Minkowski distance equal weight (KNN) using tenfold cross-validation.
\end{abstract}

Results and conclusions: The classification accuracy (ACC), sensitivity (SEN), specificity (SPE) of our proposed method yield $94.16 \%, 88.62 \%$ and $100.00 \%$, respectively. The area under the receiver operating characteristic curve (AUC) was 0.99. The experimental results indicate that the EBT algorithm with statistical textural features based on GGOs for differentiating COVID-19 from general pneumonia achieved high transferability, efficiency, specificity, sensitivity, and impressive accuracy, which is beneficial for inexperienced doctors to more accurately diagnose COVID-19 and essential for controlling the spread of the disease.

Keywords: Machine learning, Novel coronavirus pneumonia, General pneumonia, Chest CT included in the article's Creative Commons licence and your intended use is not permitted by statutory regulation or exceeds the permitted use, you will need to obtain permission directly from the copyright holder. To view a copy of this licence, visit http://creativecommons.org/ licenses/by/4.0/. The Creative Commons Public Domain Dedication waiver (http://creativecommons.org/publicdomain/zero/1.0/) applies to the data made available in this article, unless otherwise stated in a credit line to the data. 


\section{Background}

Since the first COVID-19 case was discovered in 2019, more than 9.47 million cases of novel coronavirus pneumonia have been diagnosed worldwide, with 484,249 deaths recently according to World Health Organization Coronavirus disease (COVID2019) situation report - 158. Currently, the detection of COVID-19 mainly relies on nucleic acid testing. However, many infected patients with obvious typical symptoms passed multiple nucleic acid tests but diagnosed positive in the last test [1]. The high false-negative rate results in delayed treatment and even aggravating the spread of the pandemic. On February 5, National Health Commission of the People's Republic of China launched the "Novel Coronavirus Pneumonia Diagnosis and Treatment Program (Trial Version 5)", which updated the diagnostic criteria for novel coronavirus pneumonia with adding $\mathrm{CT}$ imaging examinations as one of the main basics for clinical diagnosis of COVID-19. CT screening is considerably popular, easy to operate and sensitive to COVID-19, which is critical for both early diagnosis and pandemic control.

Nevertheless, influenza virus pneumonia and other types of pneumonia might occur in this season as well. In some aspects, especially according to clinical features, it is troublesome to differentiate COVID-19 from general pneumonia. For instance, the main manifestations of COVID-19 in the early stage were fever, fatigue, dry cough, and expiratory dyspnea while patients with general pneumonia have similar symptoms [2]. COVID-19 pneumonia places a huge burden on the health care system because of its high morbidity and mortality. Therefore, early diagnosis and isolation of GP patients and COVID-19 patients can better prevent the spread of the pandemic and optimize the allocation of medical resources. However, except for the overlapping symptoms and detection abnormalities, CT manifestations of GP and COVID19 were similar, causing instability and uncertainty for distinguishing them $[3,4]$.

Typical CT manifestations of COVID-19 patients consist of pleural indentation sign, unilateral or bilateral pulmonary ground-glass opacities, opacities with rounded morphology and patchy consolidative pulmonary opacities with the predominance in the lower lung [5-8]. GP infections have similar CT manifestations at presentation. However, COVID-19 presents more bilateral extensive GGO while GP shows more unilateral GGO or consolidation [9]. Furthermore, the other CT findings of GP and COVID-19 are difficult to observe and the areas of lungs contain large scale of insignificant extraneous parts. To avoid interference from irrelevant information and more accurately and stably identify COVID-19 from GP, GGO was cropped as the ROI and features were extracted based on ROIs. Figure 1 shows the samples of COVID-19 and GP CT images from the collected dataset.

Lin et al. proposed a deep learning model, COVNet, based on visual features from volumetric CT images to distinguish COVID-19 from community acquired pneumonia [10]. 4536 three-dimensional CT images (COVID-19: 30\%; community acquired pneumonia: 40\%; non-pneumonia: 30\%) were included in their study. U-net was applied to crop the lung region as the ROI and both 2D and 3D features were extracted by COVNet based on the ROIs. Then the features were combined and inputted to the proposed scheme for predictions. The sensitivity and specificity for detecting COVID-19 were $90 \%$ and $96 \%$ while for CAP were $87 \%$ and $92 \%$. The 


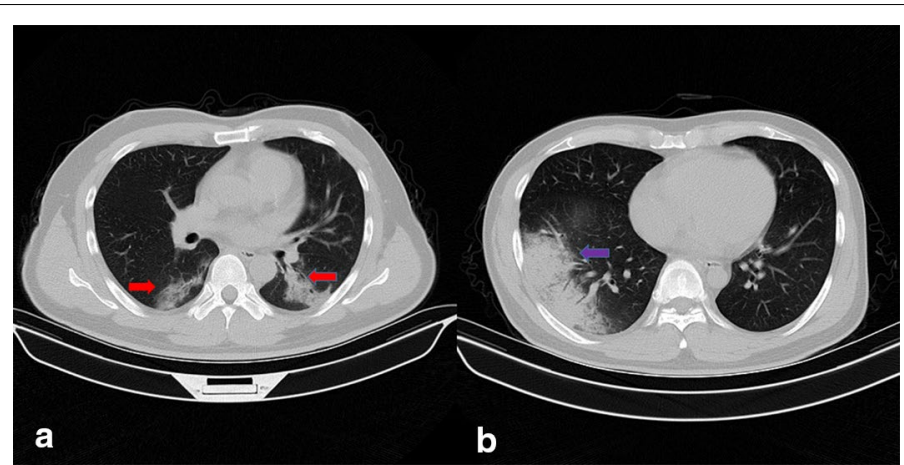

Fig. 1 Samples of COVID-19 and GP CT images. Picture $\mathbf{a}$ is the CT image of COVID-19 with bilateral GGOs while picture $\mathbf{b}$ is the CT image of GP with unilateral GGO. The red arrows point at the GGOs of COVID-19 and the blue arrow points at the GGO of GP

AUCs were 0.96 and 0.95 . However, the features learned by deep learning models are embedded in a network of millions of weights. Thus, the method lacks interpretability and transparency.

Charmaine et al. evaluated ResNet with a location-attention mechanism model for screening COVID-19 [11]. Two ResNet models were enrolled in their study. Threedimensional features were extracted by ResNet-18 and fed into ResNet-23 with location-attention mechanism in the full-connected layer for classification while ResNet without location-attention mechanism was applied as well for comparison with the proposed method. Accordingly, the results show the proposed method achieved better performance with an overall accuracy of $86.7 \%$.

Asif et al. proposed CoroNet model based on Xception architecture using X-ray images to differentiate COVID-19 from heathy, bacterial pneumonia and viral pneumonia [12]. Notably, Xception is a transfer learning model which pertained to ImageNet dataset and then retained on the collected X-ray dataset. In the proposed architecture, the classical convolution layers were replaced by convolutions with residual connections. The overall accuracy was $89.6 \%$ while average accuracy of detecting COVID-19 was 96.6\%. To test the stability and robustness, CoroNet was evaluated on the dataset prepared by Ozturk et al. [13] with an accuracy of $90 \%$.

Ozturk et al. developed DarkNet model based on the you only look once (YOLO) system to detect and classify COVID-19 [13]. Their model achieved the accuracy of $98.08 \%$ for classifying COVID-19 and non-infections and $87.02 \%$ for distinguish COVID-19 from no-findings and GP. Nevertheless, the proposed methods by Asif et al. and Ozturk et al. were based on X-ray images. X-ray screening is not sensitive to GGOs which is one of the most significant manifestations at the early stages of COVID-19. This can cause high error rate and ineffective containment of the pandemic.

Kang et al. developed a machine learning method with structured latent multi-view representation learning to diagnose COVID-19 and community acquired pneumonia [14]. In their work, V-Net was leveraged to extract lung lesions. Then, radiomic features and handcrafted features, totally 189-dimensional features, were extracted from the CT images. In the end, the proposed model yielded the best accuracy, which was $95.50 \%$. 
The sensitivity and specificity were $96.6 \%$ and $93.2 \%$. Compared with other methods in the study, the accuracy was improved by $6.1-19.9 \%$ and the sensitivity and specificity were improved by $4.61-21.22 \%$.

To our knowledge, most recent researches carried out for detecting COVID-19 are based on deep learning. However, deep learning models require a large scale of training data while initially the COIVD-19 samples are in shortage. Transfer learning might be promising method in terms of small amount of data while negative transfer may exist, for initial dataset and target domains may not relate to each other and the standards on what types of training data are sufficiently related are not clear.

Machine learning plays an unsubstitutable role in artificial intelligence with outstanding results in medical imaging classification. We developed a machine learning method using ensemble of bagged tree based on statistical texture features of CT images, particularly focusing on differentiating COVID-19 from GP, demonstrating high efficiency in the identification of COVID-19 and GP, helping to reduce misdiagnosis and control pandemic transmission.

\section{Material}

From January 2020 to March 2020, there were 73 COVID-19 cases confirmed by nucleic acid test positive and 27 general pneumonia cases enrolled in this study (age ranges from 14 to 72 years). Both COVID-19 and GP patients who had undergone chest CT scans were retrospectively reviewed by two senior radiologists. Of the COVID-19 cases, 12 patients without obvious characteristics on CT images were excluded (negative rate $16.4 \%, 12 / 73)$. Finally, 61 confirmed COIVD-19 cases and 27 general pneumonia cases were enrolled in this study.

The images were independently assessed by two radiologists. If the radiologists disagreed with each other, a senior radiologist would be invited to review the pulmonary CT images and make the final examination. All the CT images were generated from the Siemens Sensation 16-layer spiral CT (Siemens, Erlangen, Germany). The image format was Digital Imaging and Communications in Medicine (DICOM). The scan parameters were: tube voltage $120 \mathrm{kV}$; tube current automatic regulation; $1-2 \mathrm{~mm}$ cross-sectional thickness; $1-2 \mathrm{~mm}$ cross-sectional distance; scan pitch 1.3 ; and $16 \times 0.625 \mathrm{~mm}$ collimation.

\section{Results}

The proposed diagnosis method is ensemble of bagged trees based on feature combination 5 ( $T=0.11)$ including ROI delineation, feature extraction, feature selection and classification which are explicitly described in "Method" section. In this section, the results of feature selection, effectiveness of optimal feature combination 5 compared to original features, and comparison of EBT algorithm and four other classification methodologies are described. The experimental result demonstrated that the proposed COVID-19 diagnosis method outperformed other methods in terms of accuracy, sensitivity, specificity and AUC.

\section{Results of feature selection}

Table 1 and Fig. 2 show the relevance of each feature and weight curves of each feature based on ReliefF algorithm. In order to select the optimal feature combination, the 
Table 1 Relevance of each feature based on ReliefF algorithm

\begin{tabular}{llllllllll}
\hline Feature & Relevance & Feature & Relevance & Feature & Relevance & Feature & Relevance & Feature & Relevance \\
\hline 1 & 0.0421 & 8 & 0.1329 & 15 & 0.0043 & 22 & 0.1348 & 29 & 0.1250 \\
2 & 0.1036 & 9 & 0.1338 & 16 & 0.0687 & 23 & 0.1257 & 30 & 0.1025 \\
3 & 0.0050 & 10 & 0.1059 & 17 & 0.0548 & 24 & 0.1170 & 31 & 0.1490 \\
4 & 0.0178 & 11 & 0.0582 & 18 & 0.1604 & 25 & 0.1574 & 32 & 0.1250 \\
5 & 0.0032 & 12 & 0.0360 & 19 & 0.1574 & 26 & 0.2267 & 33 & 0.0389 \\
6 & 0.1434 & 13 & 0.0163 & 20 & 0.2561 & 27 & 0.2977 & 34 & 0.0963 \\
7 & 0.1036 & 14 & 0.1184 & 21 & 0.1469 & 28 & 0.2094 & & \\
\hline
\end{tabular}

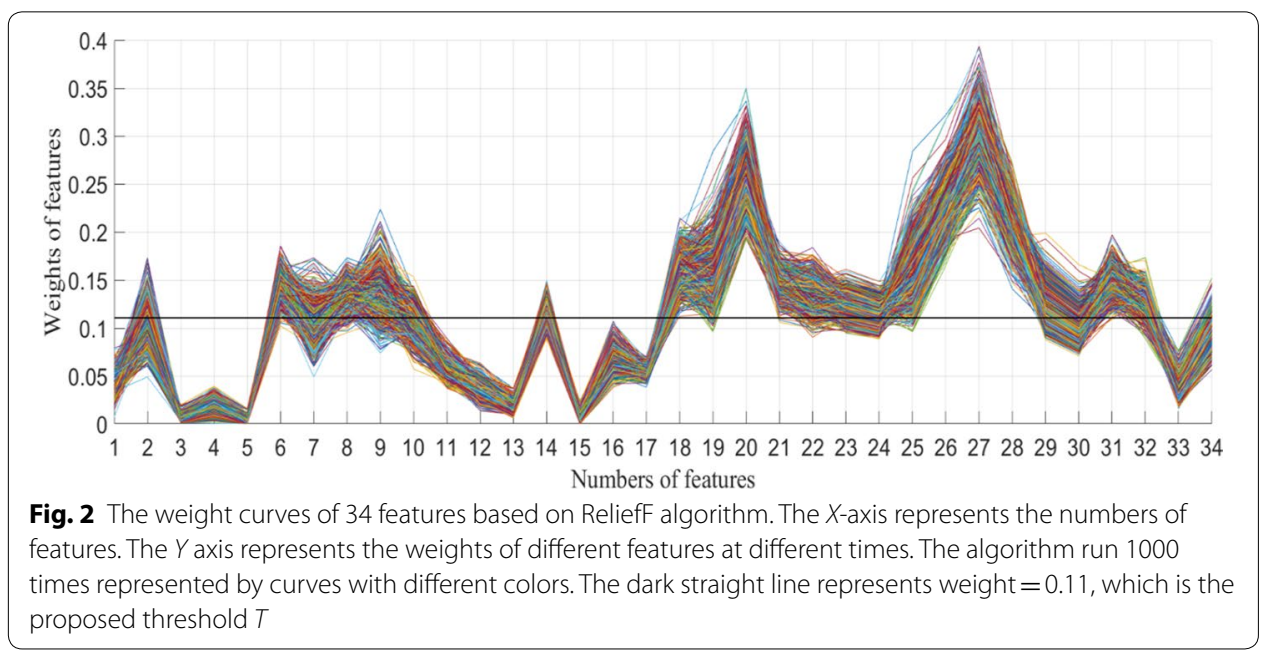

Table 2 Selected features of four combinations

\begin{tabular}{lll}
\hline Combination & $\boldsymbol{T}$ & Feature numbers \\
\hline 1 & $0.11^{\mathrm{a}}$ & $1,2,3,4,5,7,10,11,12,13,15,16,17,30,33,34$ \\
2 & $0^{\mathrm{b}}$ & All 34 features \\
3 & 0.10 & $2,6,7,8,9,10,14,18,19,20,21,22,23,24,25,26,27,28,29,30,31,32$ \\
4 & 0.12 & $6,8,9,18,19,20,21,22,23,25,26,27,28,29,31,32$ \\
5 & 0.11 & $6,8,9,14,18,19,20,21,22,23,24,25,26,27,28,29,31,32$ \\
\hline
\end{tabular}

a Select features with relevance smaller than the threshold $T$

b No feature selection was applied

proposed threshold $T$ was set to 0.11 . To justify optimization, combination $1\left(T=0.11^{*}\right)$, combination $2(T=0.12)$, and combination $3(T=0)$, combination $4(T=0.10)$ were considered to compare with combination $5(T=0.11)$. Features included according to four different $T$ values are shown in Table 2 (the corresponding feature names of the feature numbers are presented in Table 4 in "Method" section).

\section{Performance evaluation}

Table 3 shows the diagnosis performance of 5 classifiers based on 5 different feature combinations. In order to intuitively present the differences in accuracy, sensitivity and specificity of different methods using different feature combinations, we 
Table 3 Diagnosis performance based on different methods using different combinations

\begin{tabular}{cccccc}
\hline Method & Combination 1 & Combination 2 & Combination 3 & Combination 4 & Combination 5 \\
\hline DT & & & & & \\
ACC (\%) & 81.98 & 88.82 & 89.41 & 89.73 & 89.82 \\
SEN (\%) & 72.03 & 85.85 & 84.34 & 84.39 & 85.90 \\
SPE (\%) & 80.40 & 91.95 & 95.64 & 95.55 & 95.69 \\
LR & & & & & \\
ACC (\%) & 70.64 & 80.57 & 81.65 & 79.15 & 81.73 \\
SEN (\%) & 66.50 & 76.42 & 77.89 & 75.61 & 77.89 \\
SPE (\%) & 75.00 & 84.93 & 85.62 & 82.88 & 85.73 \\
SVM & & & & & \\
ACC (\%) & 81.90 & 85.32 & 85.65 & 86.07 & 86.48 \\
SEN (\%) & 76.91 & 83.58 & 82.11 & 81.30 & 81.44 \\
SPE (\%) & 87.16 & 87.16 & 89.38 & 91.10 & 88.24 \\
KNN & & & & & 82.33 \\
ACC (\%) & 77.73 & 83.23 & 85.40 & 88.32 & 96.58 \\
SEN (\%) & 69.43 & 73.98 & 79.51 & 81.79 & \\
SPE (\%) & 86.47 & 92.97 & 91.61 & 95.21 & 100.00 \\
EBT & & & & 93.16 & \\
ACC (\%) & 86.49 & 92.91 & 92.49 & 93.41 & \\
SEN (\%) & 78.37 & 86.18 & 99.69 & 99.83 & \\
SPE (\%) & 95.03 & 100.00 & & & \\
\hline
\end{tabular}

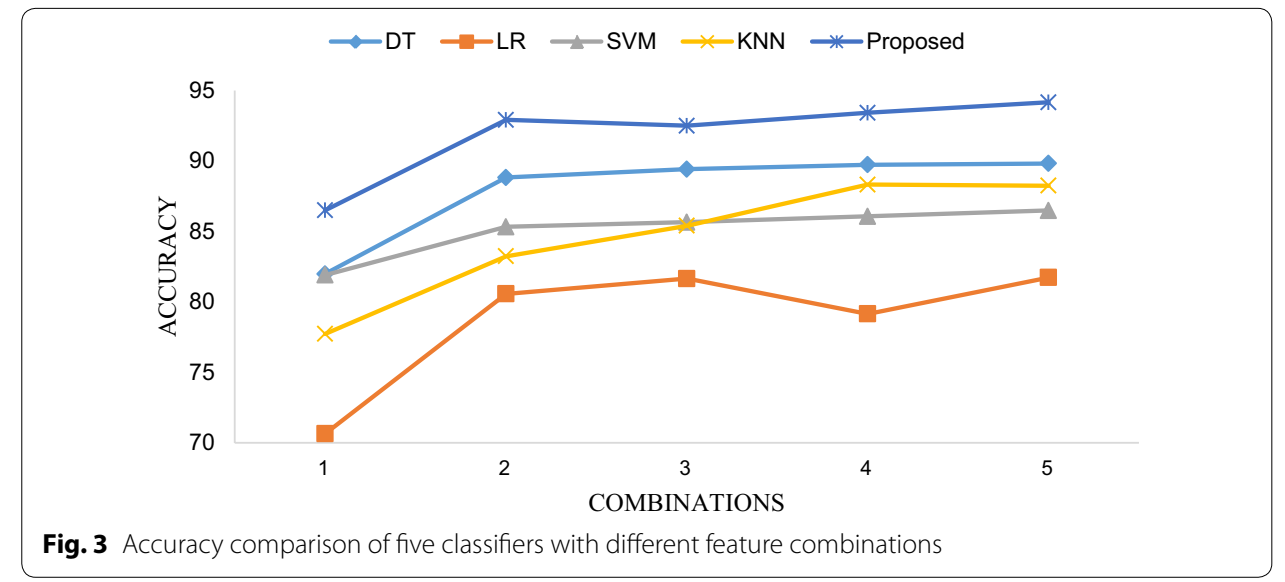

visualized them with line Fig. 3, line Fig. 4 and line Fig. 5, respectively. The receiver operating characteristic (ROC) curves of EBT algorithm and 4 other classifiers using the optimal feature combination 5 are presented in Fig. 6.

\section{Effectiveness of optimal feature combination 5 compared to original features}

Figure 3 elucidates that five classifiers using feature combination 5 achieved the highest accuracy than that obtained by other feature combinations. The measurements in the $\mathrm{X}$-axis ranging from 1 to 5 represents the sequence numbers of the feature combinations in Table 2. Figures 4 and 5 substantiate that the sensitivity and specificity of 


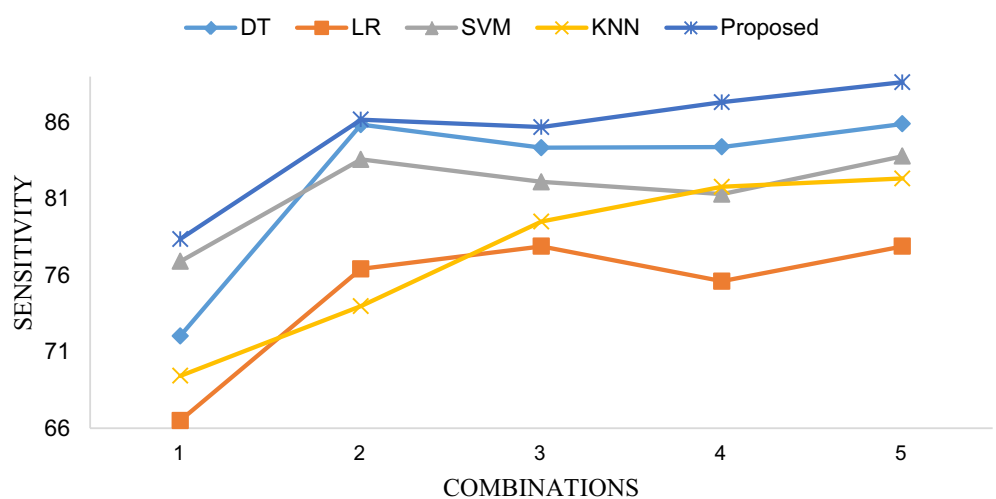

Fig. 4 Sensitivity comparison of five classifiers with different feature combinations

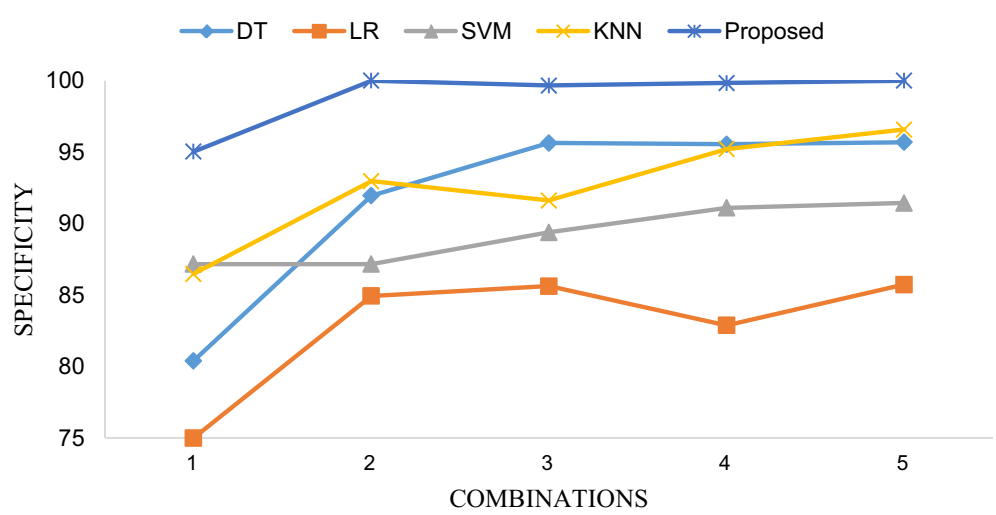

Fig. 5 Specificity comparison of five classifiers with different feature combinations

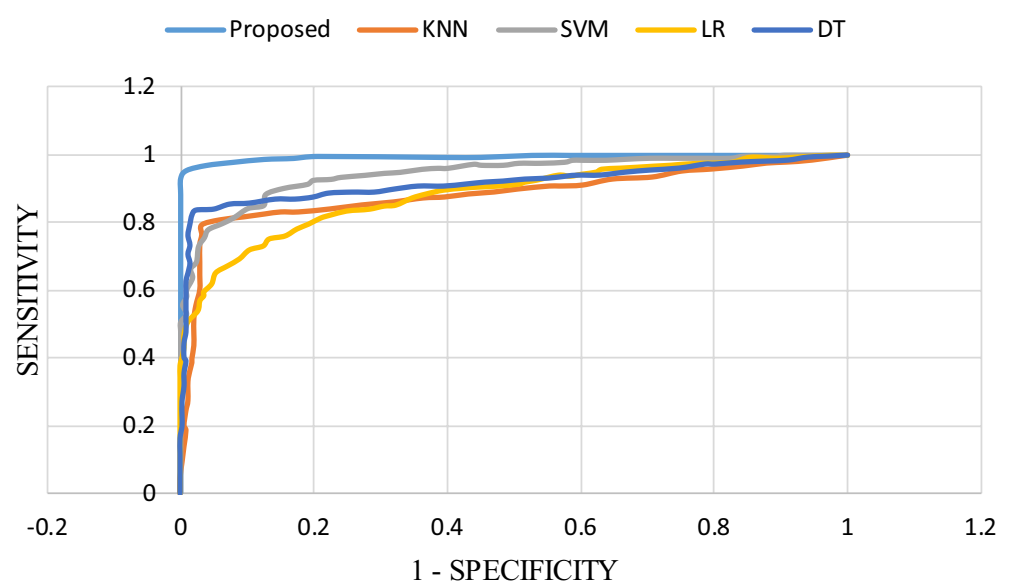

Fig. 6 Comparison of receiver operating characteristic curves for the proposed classifier, KNN, SVM, LR, and DT using feature combination 5. The receiver operating characteristic curves for the proposed EBT models had an AUC that was significantly greater than that for four other models 
the optimal feature set outperformed that of combination 2 as well as combination 1, 3 and 4 . To be noted, combination 2 contains 34 features which indicates that no feature selection was applied, which illustrates that feature selection is essential.

\section{Comparison of EBT and four other classification methodologies}

As shown in Table 3, the best result was obtained by EBT algorithm with feature combination 5, leading to accuracy, sensitivity and specificity of $94.16 \%, 88.62 \%$ and $100.00 \%$, respectively. The three line figures reveal that EBT algorithm achieved clearly better performance compared with other classification methodologies using no matter what feature combinations. Figure 6 demonstrates ROC curves of five models based on feature combination 5. And the AUCs (area under curve, AUC) of DT, LR, SVM, KNN and EBT are $0.91,0.88,0.94,0.88$, and 0.99, respectively. The EBT provided the best AUC. Therefore, the promising results validate that the proposed method can accurately and robustly differentiate COVID-19 from GP.

\section{Discussion}

The proposed diagnosis method was evaluated in terms of accuracy, sensitivity and specificity. As shown in Eqs. 2-4 in "Method" section, accuracy measures the ability of the diagnosis system to correctly detect COVID-19 and GP. Sensitivity demonstrates the proportion of correctly classified COVID-19 cases. Specificity illustrates how good the method is at identifying GP cases. As shown in Table 3, the highest accuracy, sensitivity and specificity achieved by EBT algorithm with feature combination 5 were 94.16, 88.62, and 100.00, respectively. It shows that the proposed method did better performance in detecting GP than COVID-19. To alleviate class imbalance, we did data augmentation on GP images. However, data augmentation techniques cannot increase the diversity of GP features. Although the proposed method achieved the specificity of $100.00 \%$, which suggests no GP cases were erroneously classified, there is no denying that it has the probability of over-fitting caused by shortage in GP images.

CT of COVID-19 infections presents consolidation, GGO, pulmonary fibrosis, interstitial thickening, and pleural effusion in both lungs [15-17] while CT of GP infections presents multifocal nodular opacity with a surrounding halo, diffuse patchy GGO, interlobular septal thickening, multiple ill-defined nodules and consolidation in both lungs [18]. Thus, most resent researches have proposed heterogeneous methods based on the whole lung region. For example, Wang et al. developed COVID-19Net for diagnosing COVID-19 with automatic lung segmentation of CT images using DenseNet121-FPN [19]. Notably, DenseNet121-FPN is also a transfer learning framework, which was pretrained on ImageNet dataset as well. The sensitivity and specificity of the method were $78.9 \%$ and $89.93 \%$ in the training set. In the two validation sets, the sensitivities were $80.39 \%$ and $79.35 \%$ and the specificities were $76.61 \%$ and $81.16 \%$. As mentioned previously in the background section, the deep learning method proposed by Lin et al. implemented U-net for lung segmentation [10]. It achieved the sensitivity and specificity of 90\% and 96\%. Zhang et al. used AI system with a two-stage segmentation framework to segment lung lesions and then diagnose COVID-19 [20]. The first stage of the segmentation framework was manual annotation and the second stage was DeepLabv3based backbone for lung lesion segmentation. In their work, they achieved smoother 
and clearer boundaries compared with experts. Besides, they validated their system in the dataset from outside China with $84.11 \%$ accuracy, $86.67 \%$ sensitivity, and $82.26 \%$ specificity for differentiating COVID-19 from GP. Wu et al. developed a multi-view deep learning fusion model based on the architecture of ResNet50 with threshold segmentation and morphological optimization algorithms for lung segmentation [21]. The accuracy, sensitivity and specificity of their model in the testing set were 0.760, 0.811 and 0.615 , respectively. However, compared with these studies, we did GGO segmentation instead of lung lesion segmentation. Our proposed machine learning method in combination with GGO segmentation accomplished an accuracy of $94.16 \%$ for distinguishing COVID-19 from GP. It also has a high sensitivity and specificity of $88.62 \%$ and $100.00 \%$, respectively. Therefore, we achieved better performance in diagnosing COVID-19 based on only GGOs. The results empirically validate that COVID-19 and GP can be robustly classified based on GGOs.

Despite the remarkable performance of the proposed methods, limitations still exist in our study. First of all, the ROIs were manually delineated which is rather time-consuming especially when doctors are racing against time to save lives. Also, GGOs were the exclusive segmented features of CT images of COVID-19 and GP and spending more time on ROI segmentation is apparently unworthy while the whole lung region contains irrelevant or even pernicious information for diagnosis. Hence, further study should be processed on automatically and preciously detect and segment ROIs without manual help. Finally, our established model did not determine which specific general pneumonia it was, such as viral or bacterial, mainly due to insufficient data. More data will be collected and the prognosis of GPs will be considered in our future study.

\section{Conclusions}

This study explored an ensemble of bagged tree algorithm with statistical textural features for differentiating novel coronavirus pneumonia from general pneumonia. The classification accuracy, sensitivity, and specificity of our proposed method yield $94.16 \%$, $88.62 \%$ and $100.00 \%$, respectively. It is noteworthy that compared with four other machine learning classifiers, EBT achieved consistent better performance. The results show that classifiers with feature selection excelled classifiers without feature selection by $1-5 \%$ for accuracy, $2-10 \%$ for sensitivity and $0-4 \%$ for specificity. More importantly, classifiers with feature selection take shorter time. Therefore, feature selection is beneficial for promoting the diagnosis of COVID-19 in terms of all evaluation indexes.

Furthermore, GGOs were proved to play a significant role in distinguish COVID-19 from GP, which provide reference opinions for radiologists to better diagnose COVID19. And extensive experiments will be applied on more features of COVID-19 individually and unitedly in our future work. In conclusion, the experimental results show that, as compared to other state-of-the-art works, the proposed method achieved pronouncedly superior performance with a small amount of CT images.

\section{Methods}

\section{Overview of the proposed diagnosis framework}

Machine learning algorithms integrated with statistical textural features are leveraged to differentiate COVID-19 from GP. Figure 7 illustrates the block diagram of the 


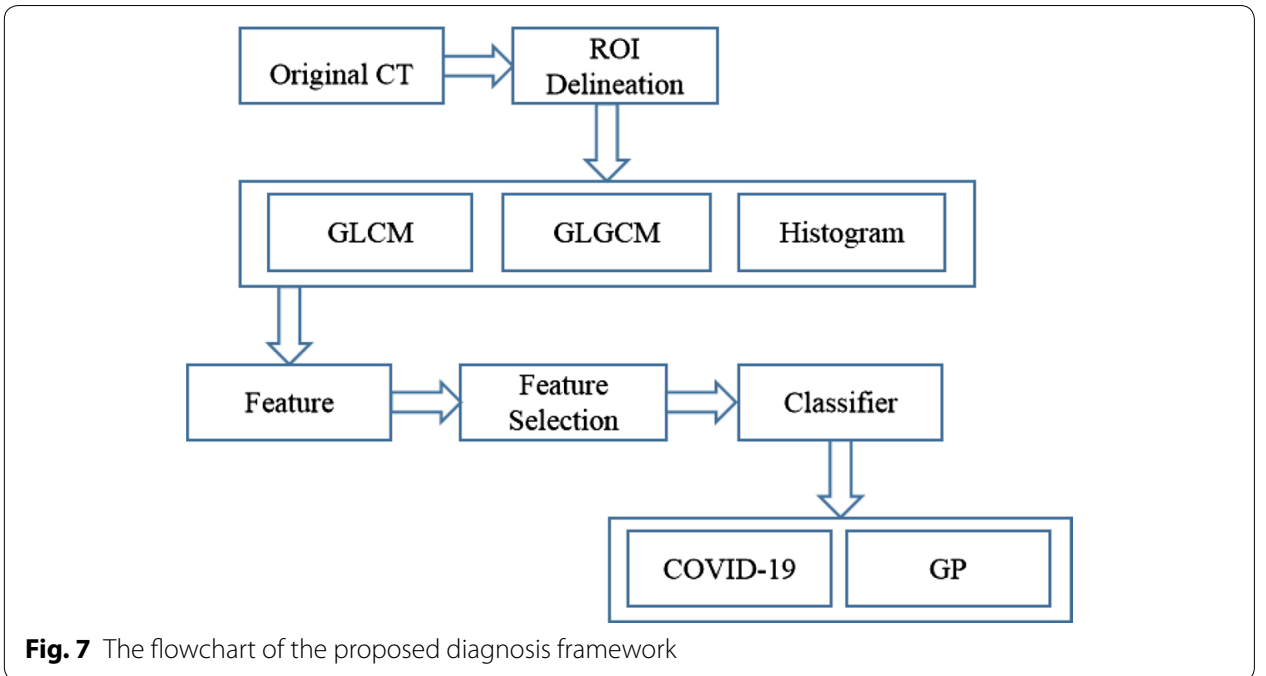

proposed diagnosis framework. After data collection, to more accurately extract features of COVID-19 and GP, manual delineation of the ROIs were performed based on GGOs. The details of ROI delineation are presented in "Delineation of ROIs" section. In the next step, 34 statistical texture features including 13 GLCM features, 15 GLGCM features and 6 histogram features were extracted from the ROIs. After that, ReliefF algorithm was used to select features for time-saving and avoiding over-fitting. As a result, five feature combinations remained while combination 5 with 18 features were classified as the proposed feature group. Details are described in the following feature selection and results part. In the last stage of diagnosis process, the selected features with labels were combined and input to five classifiers while the ensemble of bagged tree is the proposed algorithm for classification. Five classifiers with five feature combinations, respectively, were evaluated in term of accuracy, specificity, sensitivity and AUC.

The framework consists of 4 major steps: delineation of ROIs, feature extraction, feature selection, and classification. Each of the steps is described in detail in the following parts of this paper.

\section{Delineation of ROIs}

To improve the accuracy of the diagnosis method, precise segmentation of the ROIs from irrelevant parts was essential for feature extraction. Thus, GGO region, which is the main CT manifestations, was taken as ROI. The software of MRIcro 1.4 was used to extract the rectangle ROI of COVID-19 and GP. ROIs were delineated in CT images based on aforementioned GGOs. The main processes of ROI delineation are as follows: (1) a rectangular region as large as possible, which is the ROI, was delineated within GGOs and export the whole image with delineation to a PNG image; (2) PNG images were binarized to get the ROI boundary and fill the rectangular region to get the ROI template; (3) the ROI templates were used to extract the ROI in the original DICOM image; (4) the gray level of the ROI image was converted to 256 gray levels and the images were resized to $32 \times 32$ pixels. Consequently, 615 COVID-19 and 146 GP ROIs were cropped. It is apparent that COVID-19 images were four times larger than 
GP images while imbalanced data cannot reflect the true distribution of two categories, which could affect the classification performance. Thus, we rotated the GP images by $90^{\circ}, 180^{\circ}, 270^{\circ}$. Ultimately, the number of GP images was augmented to 584 . In conclusion, 1199 ROI images were enrolled for feature extraction.

\section{Feature extraction}

In this stage, a total of 34 statistical texture features were extracted from the ROI images of COVID-19 and GP as shown in Table 4, which contain 13 GLCM [22] features, 15 GLGCM [23] features and 6 histogram [24] features. GLCM and GLGCM are the predominant second-order statistical texture analysis methods to characterize the features of an image, which have been widely applied in medical image processing [25, 26]. Besides, GLCM considers the statistical and spatial relationship of the pixels in the image. It is created by calculating how often pairs of pixel with specific values and in a specified spatial relationship occur in an image. Then 13 statistical texture features are extracted based on the grey-level co-occurrence matrix. In contrast with GLCM, GLGCM captures not only gray-scale features, but also the second-order statistics of gray-level gradients while gradients indicate the information of image edge which provides significant features of an image. In addition, the histological characteristics of COVID-19 and GP can be well reflected in the gray mode, and the gray histogram is an intuitive statistical method [27]. It is a one-dimensional function of the gray level and belongs to the first-order statistical method. After obtaining all texture feature data, due to the different calculation methods of each feature, the numerical value changes in a wide range. Therefore, to facilitate calculation, all data are normalized to $[0,1]$ based on their respective dimensions, the normalized equation (1) is as follows:

$$
X *=(X-\mathrm{IN}) /(\mathrm{MAX}-\mathrm{MIN}),
$$

where $X$ is the original data of the $N_{\text {th }}$ dimension, MIN is the minimum value in the $N_{\text {th }}$ dimension, MAX is the maximum value in the $N_{\text {th }}$ dimension, $X^{*}$ is the normalized feature.

\section{Feature selection}

Feature selection plays a critical role in enhancing the performance of medical imaging classification. High-dimensional features cause over-fitting, lower accuracy, comprehension difficulty and it is rather time-consuming. Thus, feature selection is leveraged to select a subset of features, which makes the evaluation criteria reach the optimal level, from the original feature set. ReliefF algorithm is classified as a typical filter method for feature

Table 4 Description of extracted features

\begin{tabular}{|c|c|}
\hline Feature groups & Description \\
\hline GLGCM & $\begin{array}{l}\text { 1. Little gradient advantage; 2. Large gradient advantage; 3. Gray heterogeneity; 4. Gradient } \\
\text { heterogeneity; } 5 \text {. Energy; 6. Average gray; 7. Average gradient; } 8 \text {. Gray mean square error; } \\
\text { 9. Gradient mean square error; 10. Correlation; 11. Gray entropy; 12. Gradient entropy; } 13 \text {. } \\
\text { Hybrid entropy; 14. Inertia; 15. Inverse difference moment }\end{array}$ \\
\hline GLCM & $\begin{array}{l}\text { 16. Angular second moment; 17. Correlation; } 18 \text {. Entropy; } 19 . \text { Contrast; } 20 . \text { Inverse difference } \\
\text { moment; } 21 \text {. Sum average; } 22 \text {. Sum entropy; } 23 \text {. Sum variance; } 24 \text {. Variance; } 25 \text {. Dissimilar- } \\
\text { ity; 26. Inertia; 27. Difference variance; } 28 \text {. Difference entropy }\end{array}$ \\
\hline Histogram & $\begin{array}{l}\text { 29. Entropy; 30. Uniformity; 31. Mean intensity; 32. Standard deviation; 33. Kurtosis; 34. Skew- } \\
\text { ness }\end{array}$ \\
\hline
\end{tabular}


selection [28]. It calculates the weight for each feature based on the capability to identify feature value differences between nearest neighbor instance pairs. The weight of a random given feature decreases if the difference of the feature value is observed in the nearby instance of the same class (called nearest hit). Alternatively, the weight of a random given feature increases if the difference of the feature value is observed in the nearby instance of the difference class(called nearest miss). ReliefF searches for $k$-nearest hits and misses and averages their contribution to the weights of each feature [29]. Furthermore, $m$ random features will be selected and the algorithm repeated $\mathrm{n}$ times to improve reliability. After $\mathrm{n}$ iterations, divide the sum of each feature's weights by $n$. This is noted as the relevance. Features with relevance greater than a threshold $T$ are selected. Therefore, different thresholds yield different combinations. Generally, $T$ is supposed to be greater than 0 , for negative weights means negative impact on classification.

\section{Feature classification}

The ensemble of bagged tree, which is a supervised classification scheme, is regarded as the proposed classification algorithm [30]. It adopts the idea of bootstrap aggregating to enhance the stability and increase the accuracy. The training data are partitioned into several subsets by random selecting with replacement. Each subset is trained to construct independent base models. All the predictions from different models are applied to majority voting scheme. As a result, it reduces the influence of noise data and is less susceptible to over-fitting, which improves the robustness.

For comparison with the performance of the EBT algorithm, SVM, LR, DT, KNN are implemented with the same texture feature extraction methods and the same feature selection method. To superiorly identify the differences of the results, a tenfold crossvalidation strategy method is adopted. In tenfold cross-validation, the original data set is equally divided into 10 subsamples. Of the 10 subsamples, 9 subsamples are used as training set while the remaining one is taken as validation set. The process is repeated 10 times until each of the 10 subsamples is utilized as validation set. The average of the 10 results is retained as the final estimation.

\section{Statistics}

The classification metrics used included AUC, sensitivity, specificity, accuracy. Let TP (true positive) denote the number of samples belonging to class positive and correctly classified; TN (true negative) denote the number of samples belonging to class negative and correctly classified; FP (false positive) denote the number of samples not belonging to class positive but misclassified as class positive; FN (false negative) denote the number of samples not belonging to class negative but misclassified as class negative [31]. Classification accuracies are reported in terms of accuracy, sensitivity, specificity as

$$
\begin{aligned}
& \text { Accuracy }=(\mathrm{TP}+\mathrm{TN}) /(\mathrm{TP}+\mathrm{TN}+\mathrm{FP}+\mathrm{FN}), \\
& \text { Sensitivity }=\mathrm{TP} /(\mathrm{TP}+\mathrm{FN}) \\
& \text { Specificity }=\mathrm{TN} /(\mathrm{TN}+\mathrm{FP})
\end{aligned}
$$




\section{Abbreviations}

CT: Computed tomography; COVID-19: Novel coronavirus pneumonia; GP: General pneumonia; EBT: Ensemble of bagged tree; DICOM: Digital imaging and communications in medicine; kV: Kilovolt; GLCM: Gray-level co-occurrence matrix; GLGCM: Gray-level-gradient co-occurrence matrix; SVM: Support vector machine; LR: Logistic regression; DT: Decision tree; KNN: K-nearest neighbor with Minkowski distance equal weight; ROC: Receiver operating characteristic curve; AUC: Area under the receiver operating characteristic curve; TP: True positive; TN: True negative; FP: False positive; FN: False negative.

\section{Acknowledgements}

We would like to acknowledge the funding agencies for the support of the work. The content is solely the responsibility of the authors and does not necessarily represent the official views of Ruian Science and Technology Bureau.

\section{Authors' contributions}

Corresponding author:WP. CL, XW, and WP accomplished the manuscript writing, data analysis, and machine learning model developing. CL and QS accomplished data collecting, clinical expertise providing, and manuscript revising. All authors read and approved the final manuscript.

\section{Funding}

This work was supported by the funding of Ruian Science and Technology Bureau (MS2020023, MS2020025).

\section{Availability of data and materials}

The dataset analyzed during the current study was derived from the following public domain resources: https://pan. baidu.com/s/1Ux9dpa1wtquNee4hEh1OWQ, code: k23c.

\section{Ethics approval and consent to participate}

The Institutional Review Board of Ruian People's Hospital, Ruian city, Zhejiang province of China, approved the retrospective study (YJ202014), and the requirement for written informed consent was waived.

\section{Consent for publication}

All authors consent for the publication of this manuscript.

\section{Competing interests}

The authors declare that they have no competing interests.

\section{Author details}

${ }^{1}$ School of Medical Instrument and Food Engineering, University of Shanghai for Science and Technology, Shanghai 200093, China. ${ }^{2}$ College of Medical Imaging, Shanghai University of Medicine and Health Sciences, Shanghai 201318, China. ${ }^{3}$ Department of Radiology, Ruian People's Hospital, Zhejiang 325200, China. ${ }^{4}$ Department of Radiation Oncology, Chinese Academy of Medical Science (CAMS) Shenzhen Cancer Hospital, Shenzhen, 518116, China. ${ }^{5}$ Infectious Disease Department, Ruian People's Hospital, Zhejiang 325200, China.

Received: 23 May 2020 Accepted: 8 August 2020

Published online: 19 August 2020

\section{References}

1. Li D, Wang D, Dong J, Wang N, Huang H, Xu H, Xia C. False-negative results of real-time reverse-transcriptase polymerase chain reaction for severe acute respiratory syndrome coronavirus 2: role of deep-learning-based CT diagnosis and insights from two cases. Korean J Radiol. 2020;21 (4):505-8.

2. Cheng Z, Lu Y, Cao Q, Qin L, Pan Z, Yan F, Clinical Yang W. Features and chest CT manifestations of coronavirus disease, (COVID-19) in a single-center study in Shanghai, China. AJR Am J Roentgenol. 2019;2020:1-6.

3. Chung M, Bernheim A, Mei X, Zhang N, Huang M, Zeng X, Cui J, Xu W, Yang Y, Fayad ZA, Jacobi A, Li K, Li S, Shan H. CT imaging features of 2019 novel coronavirus (2019-nCoV). Radiology. 2020;295(1):202-7.

4. Li CX, Wu B, Luo F, Zhang N. Clinical Study and CT Findings of a Familial Cluster of Pneumonia with Coronavirus Disease 2019 (COVID-19). Sichuan Da Xue Xue Bao Yi Xue Ban. 2020;51 (2):155-8.

5. Dai H, Zhang X, Xia J, Zhang T, Shang Y, Huang R, Liu R, Wang D, Li M, Wu J, Xu Q, Li Y. High-resolution Chest CT features and clinical characteristics of patients infected with COVID-19 in Jiangsu. Int J Infect Dis: China; 2020.

6. Li X, Zeng W, Li X, Chen H, Shi L, Li X, Xiang H, Cao Y, Chen H, Liu C, Wang J. CT imaging changes of corona virus disease 2019(COVID-19): a multi-center study in Southwest China. J Transl Med. 2020;18(1):154.

7. Mungmungpuntipantip R, Wiwanitkit V. Clinical Features and Chest CT manifestations of coronavirus disease (COVID-19). AJR Am J Roentgenol. 2020;215:121-6.

8. Zhu T, Wang Y, Zhou S, Zhang N, Xia L. A comparative study of chest computed tomography features in young and older adults with corona virus disease (COVID-19). J Thorac Imaging. 2020;35:W97-101.

9. Yang W, Cao Q, Qin L, Wang X, Cheng Z, Pan A, Dai J, Sun Q, Zhao F, Qu J, Yan F. Clinical characteristics and imaging manifestations of the 2019 novel coronavirus disease (COVID-19): a multi-center study in Wenzhou city, Zhejiang, China. J Infect. 2020;80(4):388-93.

10. Li L, Qin L, Xu Z, Yin Y, Wang X, Kong B, Bai J, Lu Y, Fang Z, Song Q, Cao K, Liu D, Wang G, Xu Q, Fang X, Zhang S, Xia J, Xia J. Artificial Intelligence Distinguishes COVID-19 from community acquired pneumonia on chest CT. Radiology. 2020;296:E65-71.

11. Butt C, Gill J, Chun D, Babu BA. Deep learning system to screen coronavirus disease, pneumonia. Appl Intell. 2019;2020:1-7. 
12. Khan Al, Shah JL, Bhat MM. CoroNet: a deep neural network for detection and diagnosis of COVID-19 from chest x-ray images. Comput Methods Programs Biomed. 2020;196:105581.

13. Ozturk T, Talo M, Yildirim EA, Baloglu UB, Yildirim O, Rajendra Acharya U. Automated detection of COVID-19 cases using deep neural networks with X-ray images. Comput Biol Med. 2020;121:103792.

14. Kang H, Xia L, Yan F, Wan Z, Shi F, Yuan H, Jiang H, Wu D, Sui H, Zhang C, Shen D. Diagnosis of Coronavirus Disease 2019 (COVID-19) with structured latent multi-view representation learning. IEEE Trans Med Imaging 2020:39:2606-14.

15. Bai L, Gu L, Cao B, Zhai XL, Lu M, Lu Y, Liang LR, Zhang L, Gao ZF, Huang KW, Liu YM, Song SF, Wu L, Yin YD, Wang C. Clinical features of pneumonia caused by 2009 influenza A(H1N1) virus in Beijing, China. Chest. 2011;139(5):1156-64.

16. Cha MJ, Chung MJ, Lee KS, Kim TJ, Kim TS, Chong S, Han J. Clinical features and radiological findings of adenovirus pneumonia associated with progression to acute respiratory distress syndrome: a single center study in 19 adult patients. Korean J Radiol. 2016;17(6):940-9.

17. Shi H, Han X, Jiang N, Cao Y, Alwalid O, Gu J, Fan Y, Zheng C. Radiological findings from 81 patients with COVID-19 pneumonia in Wuhan, China: a descriptive study. Lancet Infect Dis. 2020;20(4):425-34.

18. Koo HJ, Lim S, Choe J, Choi SH, Sung H, Do KH. Radiographic and CT features of viral pneumonia. Radiographics. 2018;38(3):719-39.

19. Wang S, Zha Y, Li W, Wu Q, Li X, Niu M, Wang M, Qiu X, Li H, Yu H, Gong W, Bai Y, Li L, Zhu Y, Wang L, Tian J. A fully automatic deep learning system for COVID-19 diagnostic and prognostic analysis. Eur Respir J. 2020;56(2):2000775.

20. Zhang K, Liu X, Shen J, Li Z, Sang Y, Wu X, Zha Y, Liang W, Wang C, Wang K, Ye L, Gao M, Zhou Z, Li L, Wang J, Yang Z, Cai H, Xu J, Yang L, Cai W, Xu W, Wu S, Zhang W, Jiang S, Zheng L, Zhang X, Wang L, Lu L, Li J, Yin H, Wang W, Li O, Zhang C, Liang L, Wu T, Deng R, Wei K, Zhou Y, Chen T, Lau JY, Fok M, He J, Lin T, Li W, Wang G. Clinically applicable Al system for accurate diagnosis, quantitative measurements, and Prognosis of COVID-19 pneumonia using computed tomography. Cell. 2020;181(6):1423-1433.e11.

21. Wu X, Hui H, Niu M, Li L, Wang L, He B, Yang X, Li L, Li H, Tian J, Zha Y. Deep learning-based multi-view fusion model for screening 2019 novel coronavirus pneumonia: a multicentre study. Eur J Radiol. 2020;128:109041.

22. Maktabdar Oghaz M, Maarof MA, Rohani MF, Zainal A, Shaid SZM. An optimized skin texture model using gray-level co-occurrence matrix. Neural Comput Appl. 2017;9:1-19.

23. Jiang S, Mao H, Ding Z, Fu Y. Deep decision tree transfer boosting. IEEE Trans Neural Netw Learn Syst. 2019;31:383.

24. Zhang G, Ma W, Dong H, Shu J, Hou W, Guo Y, Wang M, Wei X, Ren J, Zhang J. Based on histogram analysis: ADCaqp derived from ultra-high b-Value DWI could be a non-invasive specific biomarker for rectal cancer prognosis. Sci Rep. 2020;10(1):10158.

25. Lam WC. Texture feature extraction using gray level gradient based co-occurrence matrices. IEEE Int Conf Syst. 1996;1:267-71.

26. Yang Q, Gao F, Nie Q. Analysis of rotation invariance in texture image recognition. Comput Eng Appl. 2010;46:205-7.

27. Langner T, Wikstrom J, Bjerner T, Ahlstrom H, Kullberg J. Identifying morphological indicators of aging with neural networks on large-scale whole-body MRI. IEEE Trans Med Imaging. 2019;39:430.

28. Kononenko I, Silmec E, Robnik-Sikonja M. Overcoming the myopia of inductive learning algorithms with RELIEFF. Appl Intell. 1997;7(1):39-55.

29. Urbanowicz RJ, Melissa M, La CW, Olson RS. Relief-Based Feature Selection: Introduction and Review. J Biomed Inform. 2018;7:S1532046418301400-1532046418301400.

30. Al-Barazanchi KK, Al-Neami AQ, Al-Timemy AH. Ensemble of bagged tree classifier for the diagnosis of neuromuscular disorders. In: 2017 fourth international conference on advances in biomedical engineering (ICABME), Beirut, 2017, pp. 1-4.

31. Yan Z, Zhan Y, Peng Z, Liao S, Shinagawa Y, Zhang S, Metaxas DN, Zhou XS. Multi-instance deep learning: discover discriminative local anatomies for Bodypart recognition. IEEE Trans Med Imaging. 2016;35(5):1332-43.

\section{Publisher's Note}

Springer Nature remains neutral with regard to jurisdictional claims in published maps and institutional affiliations.

Ready to submit your research? Choose BMC and benefit from:

- fast, convenient online submission

- thorough peer review by experienced researchers in your field

- rapid publication on acceptance

- support for research data, including large and complex data types

- gold Open Access which fosters wider collaboration and increased citations

- maximum visibility for your research: over $100 \mathrm{M}$ website views per year

At BMC, research is always in progress.

Learn more biomedcentral.com/submissions 